\title{
Spatial memory formation requires netrin-1 expression by neurons in the adult mammalian brain
}

\author{
Edwin W. Wong, ${ }^{1,3}$ Stephen D. Glasgow, ${ }^{1,2,3}$ Lianne J. Trigiani, ${ }^{1}$ Daryan Chitsaz, ${ }^{1}$ \\ Vladimir Rymar, ${ }^{1}$ Abbas Sadikot, ${ }^{1}$ Edward S. Ruthazer, ${ }^{1}$ Edith Hamel, ${ }^{1}$ \\ and Timothy E. Kennedy ${ }^{1,2}$ \\ ${ }^{1}$ Montréal Neurological Institute, Department of Neurology and Neurosurgery, 3801 Rue University, McGill University, Montréal, \\ Québec H3A 2B4, Canada; ${ }^{2}$ NSERC CREATE Neuroengineering Training Program, McGill University, Montréal, Québec \\ H3A 2B4, Canada
}

\begin{abstract}
Netrin-1 was initially characterized as an axon guidance molecule that is essential for normal embryonic neural development; however, many types of neurons continue to express netrin-1 in the postnatal and adult mammalian brain. Netrin-1 and the netrin receptor DCC are both enriched at synapses. In the adult hippocampus, activity-dependent secretion of netrin-1 by neurons potentiates glutamatergic synapse function, and is critical for long-term potentiation, an experimental cellular model of learning and memory. Here, we assessed the impact of neuronal expression of netrin-1 in the adult brain on behavior using tests of learning and memory. We show that adult mice exhibit impaired spatial memory following conditional deletion of netrin-1 from glutamatergic neurons in the hippocampus and neocortex. Further, we provide evidence that mice with conditional deletion of netrin-l do not display aberrant anxiety-like phenotypes and show a reduction in selfgrooming behavior. These findings reveal a critical role for netrin-1 expressed by neurons in the regulation of spatial memory formation.
\end{abstract}

Secreted chemotropic guidance cues direct axon extension during embryogenesis in the developing nervous system, yet after axon guidance is complete, many of these cues continue to be expressed by neurons and glia in the adult. Expression of guidance cues and their receptors by neurons suggests that these proteins may contribute to mature neuronal function, including synaptic plasticity underlying learning and memory (Shen and Cowan 2010). Memory consolidation is thought to involve the modification of synaptic structure and function (Roberts et al. 2010), though how guidance cues may contribute to these changes remains unclear.

Netrin-1, a canonical secreted guidance cue, is a lamininrelated protein that directs axon extension and promotes synapse formation during early development (Kennedy et al. 1994; Serafini et al. 1994; Goldman et al. 2013). The netrin receptor, deleted in colorectal cancer (DCC) (Keino-Masu et al. 1996) triggers increases in intracellular calcium, activation of RhoGTPases such as Cdc42 and Rac1, and regulates local protein synthesis (Lai Wing Sun et al. 2011; Kim and Martin 2015). Netrin-1 and DCC are highly enriched at synapses in the mature mammalian brain and DCC cofractionates with detergent-resistant components of the postsynaptic density (Horn et al. 2013). We have recently reported that netrin-1 is released at synaptic sites in response to N-methyl$\mathrm{D}$-aspartate glutamate receptor (NMDAR) activation and is critical for expression of long-term potentiation (LTP) at hippocampal Schaffer-collateral synapses, an experimental model of synaptic plasticity in the adult brain (Glasgow et al. 2018). Further, application of exogenous netrin- 1 is sufficient to trigger insertion of GluA1-containing $\alpha$-amino-3-hydroxy-5-methyl-4-isoxazolepro-

${ }^{3}$ These authors contributed equally to this work. Corresponding author: timothy.kennedy@mcgill.ca

Article is online at http://www.learnmem.org/cgi/doi/10.1101//m.049072.118. pionic acid glutamate receptors (AMPARs). Together, these findings indicate that netrin-1 participates in activity-dependent plasticity at Schaffer-collateral synapses, that netrin-1 is secreted by neurons in response to activity, and that netrin-1 is sufficient to evoke lasting synaptic potentiation (Glasgow et al. 2018). Here we report that conditional deletion of netrin-1 from principal excitatory neurons results in deficits in hippocampal-dependent spatial memory, demonstrating that netrin-1 critically regulates memory processes underlying spatial cognition.

\section{Results}

Selective deletion of netrin-1 from forebrain glutamatergic neurons impairs spatial memory

We have recently reported that deletion of netrin-1 expression from principal excitatory neurons impairs LTP in the adult hippocampus, suggesting that netrin-1 may be necessary for spatial memory formation (Glasgow et al. 2018). To test the hypothesis that netrin-1 made by glutamatergic neurons in the forebrain contributes to memory formation, we tested CaMKII-Cre/NTN $1^{f / f}$ (NTN1 cKO) and wild-type age-matched littermate controls in the hippocampus-dependent Morris water maze (MWM) task (Morris et al. 1982). For the first 3 d, the "visible" phase, mice were trained to swim to a visible platform cued by a marked object in the maze and spatial cues within the room. The "hidden" phase followed from days 4 to 8 , during which spatial cues in the room were switched and the mice were challenged to locate a hidden

(C) 2019 Wong et al. This article is distributed exclusively by Cold Spring Harbor Laboratory Press for the first 12 months after the full-issue publication date (see http://learnmem.cshlp.org/site/misc/terms.xhtml). After 12 months, it is available under a Creative Commons License (Attribution-NonCommercial 4.0 International), as described at http://creativecommons.org/licenses/by-nc/4.0/. 
platform placed in a different quadrant of the maze with the visible cue removed. All animals performed similarly with regard to escape latency to reach the platform, indicating intact sensory and motor functions (Fig. 1A). The observed improvement in performance across training days is consistent with the formation of a cognitive spatial map (Brody and Holtzman 2006). Twenty-four hours following the final training session (day 8), the platform was removed. We observed no significant differences in the swimming speed of NTN1 cKO and cre-negative wild-type littermate mice (Fig. 1B). However, adult NTN1 cKO mice made significantly fewer crosses over the location of the removed platform during the probe trial, spent less time within the target quadrant, and traveled less distance within the target quadrant compared to control wild-type littermate mice (Fig. 1C-E). These findings provide evidence that neuronal expression of netrin-1 is critical for spatial memory consolidation and precision.

While the impairments described above suggest a deficit in spatial memory in NTN1 cKO mice, performance in the MWM can also engage the encoding and retrieval of emotionally aversive training events (D'Hooge and De Deyn 2001). To test whether neuronal netrin-1 expression is necessary for hippocampal-dependent spatial memory consolidation, we tested NTN1 cKO and agematched control littermate mice using the novel object place recognition (NOPR) task (Fig. 2A). We observed no significant differences in total exploration time between NTN1 cKO and age-matched wild-type littermate control mice (Fig. 2B). In contrast, we observed significant decreases in discrimination ratio and reduced investigative ratios in NTN1 cKO mice compared to control littermates (Fig. 2C,D). Control littermates also showed an expected higher interaction count for the novel placed object compared to the unmoved, familiar placed object during the
Choice Phase, while NTN1 cKO mice showed no differences between the two objects (Fig. 2E). Together, these findings strongly implicate a critical role for neuronal expression of netrin-1 in spatial memory.

\section{Selective deletion of netrin-1 from forebrain glutamatergic neurons reduces self-grooming but does not elicit abnormal anxiety-like behavior}

Though widely used as a measure of spatial memory, the MWM has been reported to induce anxiogenic confounding behaviors due to its reliance on retrieval of emotionally aversive memories associated with the task (Harrison et al. 2009). Further, stress hormones can be elevated in rodents when assessed in the MWM, which may disturb or influence memory (Vogel-Ciernia and Wood 2014). To determine if mice lacking netrin-1 in excitatory neurons exhibited phenotypes that could impact performance in the MWM, we tested NTN1 cKO and age-matched littermate controls in an open field test to measure possible anxiety and motor abnormalities (Seibenhener and Wooten 2015). Analysis of the open field test relies on a rodent's innate exploratory behavior coupled with aversion to open spaces, and can be used to assess for hyper- or hypo-locomotor activity (Crawley 1985). A greater preference to travel along the boundary of the box compared to the center region is interpreted as increased anxiety. Mice were first habituated to the open field for $15 \mathrm{~min}$ before a subsequent 75 -min experimental trial with movement trajectories recorded via an overhead camera (Fig. 3A). Locomotor activity was expressed as distance traveled in successive 3-min bins over the course of the experimental trial (Fig. 3B). No significant differences in locomotor activity were detected between NTN1 cKO and controls across the duration of the
A
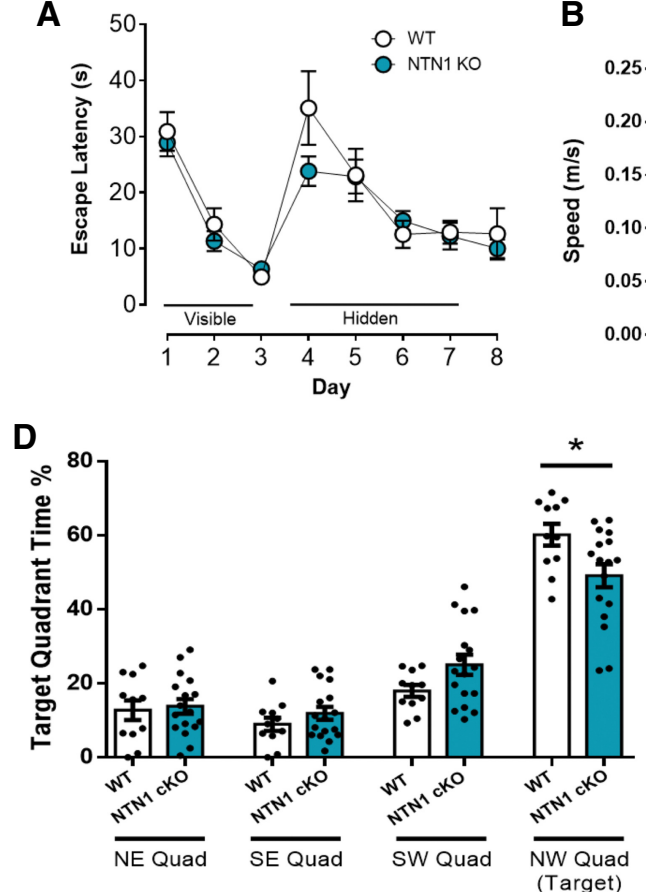

B

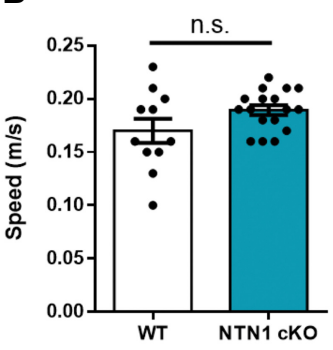

E

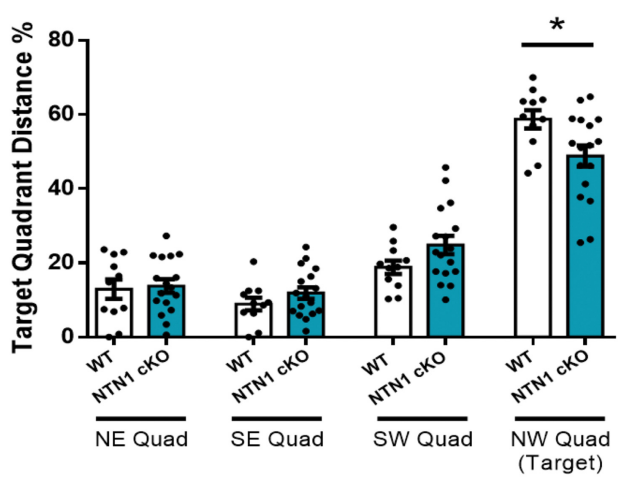

C

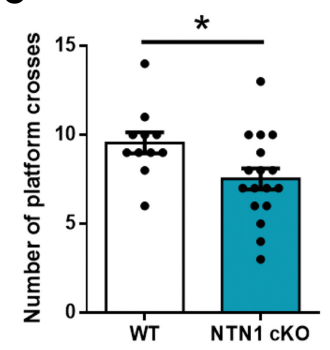

Figure 1. Mice with conditional deletion of netrin-1 from glutamatergic neurons in the forebrain exhibit spatial memory deficits in the Morris water maze. $(A)$ Both wild-type and NTN1 cKO mice showed no differences in performance during the training phases (WT: $n=11$, NTN1 cKO: $n=17$; two-way repeated-measures ANOVA, Bonferroni's post-hoc test). ( $B-E$ ) No differences were observed in speed between genotypes ( $B$; WT: $0.1700 \pm$ 0.011 , NTN1 cKO: $0.1894 \pm 0.0046, P=0.083)$. NTN1 cKO mice (blue bars) made fewer passes over platform location $(C ;$ WT: $9.545 \pm 0.593$, NTN1 CKO: $7.529 \pm 0.59, P=0.031)$, spent proportionally less time ( $D ;$ WT: $58.75 \pm 2.47$, NTN1 cKO: $48.82 \pm 2.89, P=0.022)$, and traveled significantly less distance ( $E ;$ WT: $60.18 \pm 2.93$, NTN1 cKO: $49.11 \pm 3.13, P=0.023$ ) in the target quadrant compared to control mice (white bars). All comparisons were performed with two-tailed independent $t$-test where $\left(^{*}\right) P<0.05$. Data are shown as mean \pm SEM. 
A

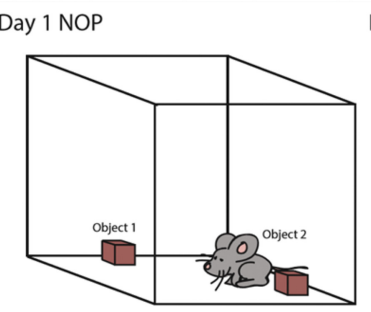

Day 2 NOP

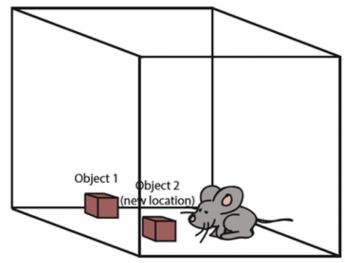

C

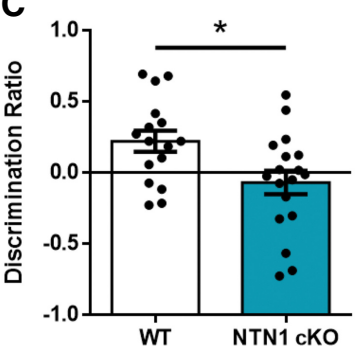

D

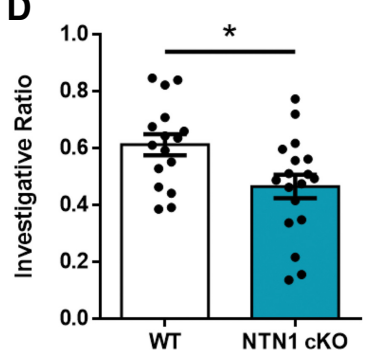

B

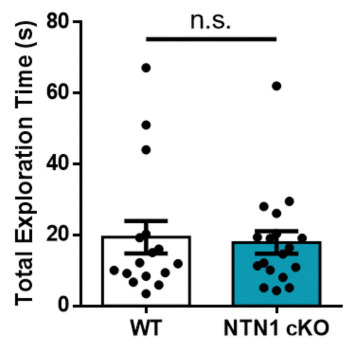

E

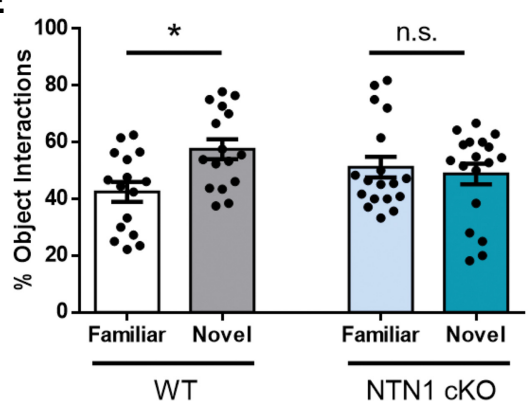

Figure 2. NTN1 CKO mice exhibit spatial memory deficits in the novel object place recognition (NOPR) test. (A) Schematic of the NOPR test. (B) No significant differences were observed in the total exploration time for either object (WT: $n=16,19.36 \pm 4.58$, NTN1 cKO: $n=18,17.86 \pm 3.17, P=$ 0.79). ( $C, D)$ NTN1 CKO scored significant less on the discrimination ratio $(C ;$ WT: $0.223 \pm 0.074$, NTN1 $C K O:-0.067 \pm 0.083, P=0.014)$ and investigative ratio $(D ; W T: 0.6137 \pm 0.037$, NTN1 CKO: $0.4664 \pm 0.041, P=0.013)$ during the Choice Phase. $(E)$ Wild-type mice displayed significantly more interactions with the novel placed object compared to the familiar object but not NTN1 cKO animals (WT Familiar: 42.46 \pm 3.47 , WT Novel: 57.54 \pm 3.47 , NTN1 cKO Familiar: $51.21 \pm 3.71$, NTN1 cKO Novel: $48.79 \pm 3.71$, Genotype $X$ Choice interaction: $F_{3,64}=2.82, P<0.05$, one-way ANOVA; pairwise comparisons, WT Familiar versus WT Novel: $P<0.028$, NTN1 CKO Familiar versus NTN1 CKO Novel: $P<0.96)$. All pairwise comparisons were performed with Tukey's multiple comparison test. All data are shown as mean \pm SEM.

test. Additionally, no differences were observed between genotypes in the distance traveled along the border and center of the open field box (Fig. 3C). Moreover, NTN1 cKO and controls did not differ in the total distance traveled (Fig. 3D) or total activity counts (Fig. 3E). These findings indicate that deletion of netrin-1 from glutamatergic neurons in the forebrain does not elicit gross-motor impairments or alteration of anxiogenic behaviors. This absence of changes in anxiety is also consistent with the lack of a difference between NTN1 cKO and control mice during the first $3 \mathrm{~d}$ of visible platform training in the MWM.

Grooming behavior in rodents is an indirect measure of several behavioral phenomena such as motor sequencing and patterning, motivation, and anxiety (Kalueff et al. 2016) that are dependent on multiple brain regions including the limbic system and forebrain cortical regions. We therefore investigated whether the deletion of netrin-1 in NTN1 cKO mice might result in abnormalities related to repetitive grooming behaviors. Interestingly, NTN1 cKO mice displayed a significant reduction in the amount of time spent grooming relative to controls (Fig. 3F).

\section{Selective deletion of netrin-1 from forebrain glutamatergic neurons does not impair novelty-seeking behavior}

The NOPR test is commonly used as an alternative measure of hippocampal-dependent spatial memory that is less stressful than the MWM (Bannerman et al. 2014). However, due to its reliance on a rodent's innate preference for novelty, a potential confounding aspect is that NTN1 cKO mice may exhibit deficits in novelty-seeking behavior, which may contribute to their poor performance in the NOPR task. To assess whether deletion of netrin-1 from forebrain glutamatergic neurons impairs novelty-seeking, we assessed NTN1 cKO and control age-matched wild-type littermates using a T-maze spontaneous alternation test (Fig. 4A). This task is based on the innate motivation of rodents to explore novel environments. Mice were placed in the "starting arm" and allowed to choose a "goal arm" (i.e., left or right arm). Following a 30-sec delay, animals were placed back in the "starting arm" and again allowed to choose a "goal arm." No significant difference was detected between genotypes in the spontaneous alternation task (Fig. 4B), indicating that netrin-1 deletion in the NTN1 cKO mice does not disrupt preference for novelty.

\section{Discussion}

The present study examined the behavioral consequences of conditional deletion of netrin- 1 from forebrain glutamatergic neurons in adult mice. Previous studies indicate that netrin-1 and its receptor DCC are enriched at mature synapses, suggesting a role in synaptic transmission (Horn et al. 2013). Recent findings indicate that conditional deletion of netrin- 1 from principal excitatory hippocampal neurons results in severe impairment of LTP, and that netrin- 1 secreted by neurons potently regulates synaptic transmission and plasticity in the adult hippocampus (Glasgow et al. 2018), a brain region critical for the consolidation of spatial memory (Nakazawa et al. 2004). Deficits in synaptic plasticity at Schaffer-collateral synapses are associated with impairments in spatial memory retrieval and disruption of spatial representations (Moser et al. 1998; Brun et al. 2001). We hypothesized that deletion of netrin-1 expression in principal excitatory neurons would selectively impair spatial memory performance. To assess for a role in spatial memory, we tested the performance of mice conditionally lacking netrin-1 expression in principal excitatory forebrain and hippocampal neurons (NTN1 cKO) in two hippocampal-dependent spatial memory tests: MWM and NOPR. Our findings demonstrated impaired spatial memory function in NTN1 cKO mice compared to age-matched control wild-type 
A

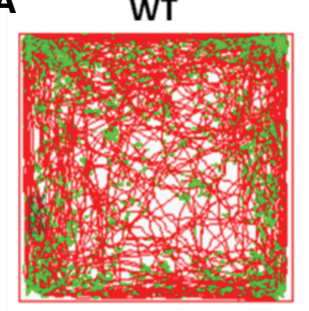

cKO

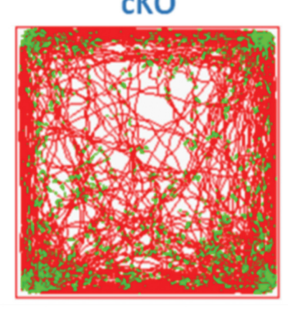

B

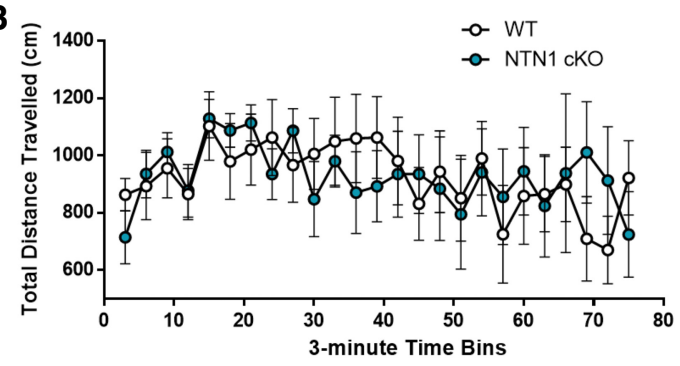

C

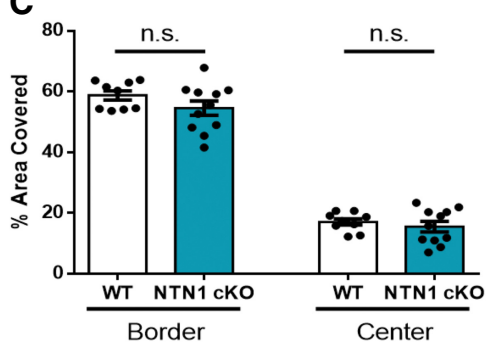

D

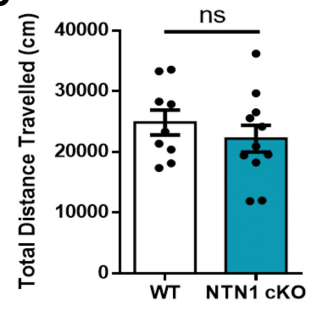

E

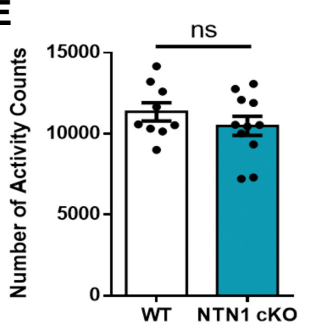

$F$

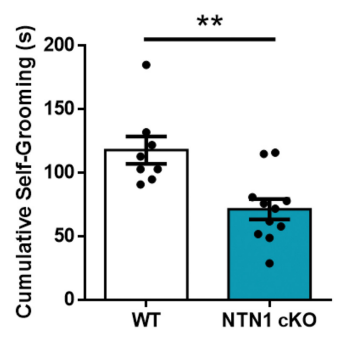

Figure 3. NTN1 cKO mice do not exhibit abnormal anxiety-like behaviors but show reduced self-grooming. ( $A$ ) The trajectories traveled by the mice in the open field test were recorded by an overhead video-based tracking system. $(B)$ Locomotor activity expressed as distance traveled in successive 3-min bins in the open field test (75 min total). No differences were observed between wild-type and NTN1 cKO mice for all time bins (WT: $n=9$, NTN1 cKO: $n=$ $11, P>0.05$ for all time bins). (C) No significant differences were observed between genotypes for distance covered either within the border close to the walls of the chamber (WT: $58.75 \pm 1.51$, NTN1 cKO: $54.55 \pm 2.38, P=0.17$ ) or within the center of the field (WT: $17.06 \pm 1.05, \mathrm{NTN} 1 \mathrm{cKO}$ : $15.49 \pm 1.73, P=0.47)$. Genotypes did not differ in the total distance traveled ( $D ;$ WT: $24,868 \pm 2053, N T N 1 \mathrm{cKO}: 22,217 \pm 2190, P=0.40)$ and the total counts of activity ( $E$; WT: $11,357 \pm 558$, NTN1 cKO: $10,482 \pm 594, P=0.30)$ through the duration of the test. $(F)$ NTN1 cKO mice displayed significant reduction in spontaneous self-grooming (WT: $n=8,118.0 \pm 10.72$, NTN1 cKO: $n=11,71.64 \pm 7.97, P=0.004$ ). All comparisons were performed with twotailed independent $t$-tests unless otherwise specified. Data are shown as mean $\pm \mathrm{SEM}$.

littermates. Among the tests run, the observed deficits were specific to spatial memory, and no abnormalities in motor performance were detected in NTN1 cKO mice. However, netrin-1 and DCC are widely expressed throughout the hippocampus and neocortex (Volenec et al. 1997; Horn et al. 2013; Cembrowski et al. 2016; Glasgow et al. 2018), and we do not rule out potential contributions to other forms of learning and memory. Taken together, the present study reveals a critical role for netrin-1 expression by neurons in the regulation of the synaptic mechanisms that contribute to spatial memory.

\section{Conditional deletion of netrin-1 does not influence novelty-seeking}

The CA3 region of the hippocampus has been proposed to serve as a comparator of novel stimuli, and hippocampal processing contributes to novelty detection in rodents (Vinogradova 2001; Kumaran and Maguire 2009). Disruption of the CA3 Schaffer collaterals synaptic inputs to CA1 pyramidal neurons impairs both spatial memory and novelty-seeking behavior (Vago and Kesner 2008). Since the NOPR task relies on a rodent's intrinsic preference for novelty, alterations in the mechanisms underlying noveltyseeking might contribute to impaired behavioral performance in NTN1 cKO mice. However, we observed no differences in spontaneous alternation on a T-maze task as an assessment of noveltyseeking. This indicates that loss of netrin-1 in forebrain glutamatergic neurons does not disrupt novelty detection, and supports the conclusion that the behavioral deficits observed are due to spatial memory dysfunction.

DCC haploinsufficient mice display blunted motor responses to amphetamine (Flores et al. 2005; Kim et al. 2013). These findings suggest that netrin-1 and DCC may influence the neural control of motor behavior in rodents. While we did not detect a significant perturbation of locomotor activity in NTN1 cKO mice in the open field test or in the MWM, we did identify a decrease in spontaneous self-grooming by NTN1 cKO mice. Self-grooming is a highly evolutionary conserved behavior that involves complex patterning of motor movements and is dependent on multiple brain regions, including the neocortex, striatum, and hypothalamus (Kalueff et al. 2016). Interestingly, disruption of glutamatergic synaptic activity in the neocortex has been reported to affect self-grooming (Aida et al. 2015; Kalueff et al. 2016). Although the specific neural mechanisms that underlie altered self-grooming remain unclear, enhanced cellular and synaptic excitability can
A

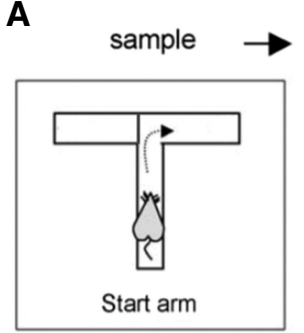

delay
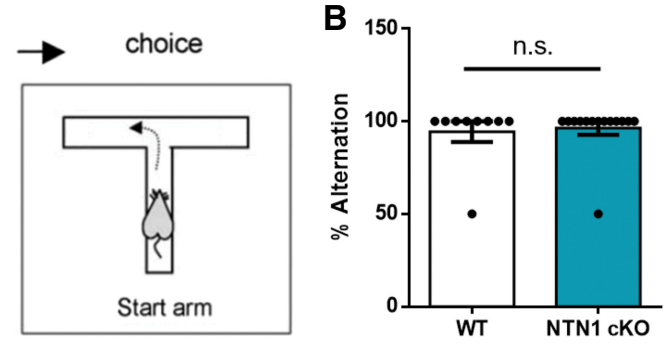

Figure 4. Mice with conditional forebrain netrin-1 deletion do not exhibit deficits in novelty preference. $(A)$ Schematic representation of the spontaneous alternation T-maze test. (B) No differences were observed between age-matched wild-type and NTN1 CKO mice in the spontaneous alternation T-maze (WT: $n=9,94.44 \pm 5.56$, NTN1 cKO: $n=14,96.43 \pm 3.57, P>0.99$, two-tailed Mann-Whitney test). Data shown as mean \pm SEM. 
increase repetitive behaviors. For example, mice lacking the astrocyte-specific glutamate transporter, GLT1, display aberrant excitatory transmission at corticostriatal synapses, along with increased self-grooming. In contrast, administration of an NMDAR antagonist, memantine, ameliorated the pathological selfgrooming (Aida et al. 2015). We have previously demonstrated that netrin-1 alters NMDAR-dependent LTP, suggesting that deletion of netrin-1 may modify NMDAR function (Glasgow et al. 2018). As such, the observed reduced grooming may be a consequence of synaptic modulation mediated by NMDAR activation and changes in synaptic excitability due to a loss of netrin-1 expression.

\section{Netrin-1 and spatial memory consolidation}

Netrin-1 signaling through DCC activates kinases involved in the regulation of LTP (Lai Wing Sun et al. 2011; Park et al. 2016; Glasgow et al. 2018; Incontro et al. 2018) and conditional deletion of netrin-1 or DCC from principal excitatory neurons severely attenuates an NMDAR-dependent form of LTP (Horn et al. 2013; Glasgow et al. 2018). In the developing nervous system, DCC directs cell motility and axon guidance through the activation of phospholipase $\mathrm{C}-\gamma$, as well as regulating intracellular calcium, focal adhesion kinase, and local protein synthesis (Lai Wing Sun et al. 2011; Kang et al. 2018). During postnatal maturation in mice, netrin-1 and DCC are synapse enriched proteins that promote adhesion, locally reorganize the actin cytoskeleton and enrich for synaptic proteins, such as PSD95, via Src family kinase and mTOR-dependent mechanisms (Goldman et al. 2013). In the mature brain, NMDARs activate signaling pathways critical for LTP, including triggering the exocytosis of netrin-1 (Lynch et al. 1983; Glasgow et al. 2018). This local release of netrin-1 results in DCC-dependent and CaMKII-mediated recruitment of synaptic GluA1-containing AMPARs to facilitate synaptic transmission. Together, these findings indicate that netrin-1 signaling via DCC is critical to activate the mechanisms that underlie the long-term changes in synaptic strength associated with LTP.

Changes in synaptic strength are necessary for the formation of place cells, a subset of CA1 pyramidal neurons whose activity is linked with contextualized location-specific firing. Their activity is markedly elevated when an animal's head is in specific regions of the environment ("place fields") and virtually silent outside of these regions (O'Keefe and Dostrovsky 1971). The activity of a single place cell is correlated with cellular activity in adjacent place fields, which generates a spatial map of the environment $\left(\mathrm{O}^{\prime} \mathrm{Neill}\right.$ et al. 2006). Importantly, replay of place cell activity between two synaptically connected CA1 pyramidal neurons can induce NMDAR-dependent LTP (Isaac et al. 2009). In the intact animal, consolidation of spatial memory may be due to temporally compressed replay of place cell sequences, resulting in high-frequency stimulation of synaptic inputs onto CA1 pyramidal neurons that triggers LTP-like synaptic consolidation (Nakazawa et al. 2004; Sadowski et al. 2016). Replay of place cell sequences is dependent on activation of NMDARs (Silva et al. 2015), which in turn can evoke netrin-1 release and activation of downstream signaling mechanisms involved in synaptic consolidation. Consequently, a lack of netrin-1 may disrupt the consolidation of spatial information; however, it remains unclear how netrin-1 might contribute to place cell formation and stabilization, as well as the network synchronization required for memory function.

Synchronous network activity plays a critical role in coordinating neuronal activity (Buzsáki 2002). Electrical field activity recorded in the hippocampus is dominated by $\theta$-frequency $(5-10 \mathrm{~Hz})$ oscillations, large amplitude sinusoidal-like waveforms that are most prominent during periods of active exploration and rapid-eye movement (REM) sleep. Consistent with a critical role in synaptic plasticity, REM sleep $\theta$ activity and place cell replay are necessary to encode previously acquired memories (Louie and Wilson 2001; Boyce et al. 2016). Oscillatory activity facilitates the coordination of synaptic inputs onto CA1 pyramidal neurons to increase postsynaptic depolarization (Montgomery et al. 2008). We predict this will promote netrin-1 exocytosis and thereby modify the synaptic connections that underlie spatial memory; however, further studies are required to determine how netrin-1 modulates network-level activity and plasticity to influence the formation of spatial memories.

\section{Materials and Methods}

\section{Animals}

All procedures were performed in accordance with the Canadian Council on Animal Care guidelines for the use of animals in research and approved by the Montreal Neurological Institute Animal Care Committee. T29-1 CaMKII $\alpha$-Cre mice (Tsien et al. 1996) were obtained from The Jackson Laboratory (Bar Harbor, ME, USA), maintained on a C57BL/6 genetic background and crossed with mice homozygous for the floxed netrin-1 allele, netrin- $1^{f / f}\left(\mathrm{NTN1} 1^{f / f}\right)$, also maintained on a C57BL/ 6 genetic background (Glasgow et al. 2018). Cre recombinase is first expressed $\sim 2.5 \mathrm{wk}$ postnatally, with expression throughout the forebrain but limited to glutamatergic neurons, including all hippocampal subfields by 1 mo of age. Importantly, cre expression occurs well after the establishment of major axon tracts (Tsien et al. 1996; Horn et al. 2013). Previous work has demonstrated that netrin-1 protein levels in CaMKII $\alpha$-Cre-NTN1 $1^{f / f}$ (NTN1 cKO) mice are significantly reduced by 3 mo of age; therefore, all experiments were performed with mice between 3 and 9 mo old (Glasgow et al. 2018). We failed to observe significant effects of sex or age on behavioral measures, and therefore all data were pooled. Age-matched wild-type littermates were cre-negative NTN1 1 ff

\section{Morris water maze}

Spatial memory was evaluated in the MWM, as described previously (Tong et al. 2012). NTN1 cKO and wild-type age-matched cre-negative littermates were trained on a modified 9-d protocol to assess for hippocampal-dependent spatial memory (Clark and Martin 2005). Briefly, mice were first trained for $3 \mathrm{~d}$ in a "visible" familiarization phase, during which a marked object was placed on the platform in a circular pool $(140 \mathrm{~cm}$ diameter) filled with opaque, cold water $\left(18 \pm 1^{\circ} \mathrm{C}\right)$, with visual cues located on the walls of the room equidistant above the water level. This was followed by five successive days of "hidden" platform testing where mice had to escape onto the platform relocated to a different quadrant within the pool and submerged $\sim 1 \mathrm{~cm}$ under the water surface, with spatial visual cues repositioned in the room. On day 1 , animals that failed to locate the platform during the trial were guided to the platform and allowed to observe the visual cues for $10 \mathrm{sec}$. Mice that demonstrated consistent visual or motor abnormalities during the familiarization phase were removed from the analysis. Mice were randomly placed in a different area of the pool between training trials. Each trial lasted a maximum of $1 \mathrm{~min}$. Twenty-four hours after the last training session, spatial memory was assessed using a probe trial in which the platform was removed. Escape latency during training, and automated unbiased analysis of movements during the probe trial, were measured using 2020 Plus tracking system and Water 2020 software (Ganz FC62D camera, HVS image).

\section{Novel object placement}

Mice were trained and tested using the novel object place recognition (NOPR) test as a noninvasive measure of hippocampaldependent spatial memory, which lasted a total of $3 \mathrm{~d}$ (Boyce et al. 2016). On day 1 , mice were habituated to the square testing chamber $(50 \times 36 \mathrm{~cm}, 26-\mathrm{cm}$ high wall $)$ for $5 \mathrm{~min}$ without any added objects. On day 2, during the "Sample Phase," mice were 
exposed to two identical objects for $5 \mathrm{~min}$ in two separate training sessions that took place $4 \mathrm{~h}$ apart. Twenty-four hours following the last training session, one of the objects was moved to a novel location in the square chamber and the mice were provided $5 \mathrm{~min}$ to explore both objects. An overhead camera recorded the mice in the square chamber throughout training and testing, and exploration time was measured by an experimenter blind to the genotypes. Objects and the test chamber were cleaned with $70 \%$ ethanol between trials to remove any olfactory cues.

Exploration times were calculated as the total time in the probe trial the animal investigated both objects (within one body length from an object with head pointed toward the object). Investigative ratios were calculated as the time spent exploring the novel placed object divided by the total time spent exploring both objects during the probe trial. Discrimination ratios were calculated as the difference between the time exploring the novel placed object and the time exploring the familiar placed object divided by the total time spent exploring both objects during the probe trial. Percentages of interactions with the novel placed object were reported as the number of contacts with the novel placed object divided by the total counts for both objects.

\section{Open field test}

Mice were placed individually into clean open white square chambers $(50 \times 50 \mathrm{~cm}, 34-\mathrm{cm}$ high wall) for a total of $90 \mathrm{~min}$. Animal activity in the box was monitored using an infrared photobeam tracking system (VideoTrack, ViewPoint Life Sciences). Mice were habituated inside the open field for the first $15 \mathrm{~min}$, followed by the experimental trial, which lasted $75 \mathrm{~min}$ (Seibenhener and Wooten 2015). Total distance traveled, number of activity counts (i.e., initiation of movements), and time traveled were measured. The number of beam breaks were recorded every $3 \mathrm{~min}$. Image analysis of the distance spent in the border and center regions of the boxes was performed using Fiji software (Schindelin et al. 2012).

\section{Spontaneous alternation T-maze}

The spontaneous alternation T-maze relies on a rodent's attraction to explore novel environments and was used to assess for possible impairments in novelty-seeking behavior (Deacon and Rawlins 2006). Mice were initially placed in the "start arm" of the T-maze (arms $30 \times 10 \mathrm{~cm}, 20-\mathrm{cm}$ high walls) and could subsequently choose a "goal arm" (Sample Phase). The animal was then confined within the chosen arm for $30 \mathrm{sec}$. A choice was defined as all four paws inside the arm. Following this, both the mouse and door were removed simultaneously, and the animal placed in the "start arm" facing away from the goal arms (Choice Phase). The animal was then allowed to again choose between the two open "goal arms" with the choice recorded. Based on the novelty of the previously unchosen arm, a correct choice occurs when the mouse alternates between arms, and an incorrect choice occurs when they animal does not alternate between arms. Two test trials, $24 \mathrm{~h}$ apart, were performed for each animal. For quantification, a score of either $100 \%$ or $0 \%$ was given to each mouse, and the average score for both trials per animal was calculated.

\section{Spontaneous self-grooming}

Spontaneous self-grooming is commonly used as a measure of repetitive behavior and motor coordination. Each mouse was individually placed in a novel mouse cage with a thin $(1 \mathrm{~cm})$ layer of bedding to reduce neophobia and prevent digging as a possible competing behavior (Silverman et al. 2010). Following a 5-min habituation period in the test cage, each mouse was scored for the total time spent grooming all body regions for $10 \mathrm{~min}$ (McFarlane et al. 2008). The observer sat approximately $2 \mathrm{~m}$ from the test cage and was blind to genotype.

\section{Statistical analyses}

Statistical analyses on parametric data were assessed using two-way repeated-measures analysis of variance (ANOVA) followed by Bonferroni's post-hoc test, one-way ANOVA followed by Tukey's pairwise comparison test, and independent $t$-tests where appropriate. Analyses on nonparametric data were assessed using two-tailed Mann-Whitney test. Normality, homoscedasticity, and outlier tests were performed on all data sets. Statistical analyses were performed using GraphPad Prism. All data shown are presented as mean \pm SEM, with statistical significance accepted as $P<0.05$ using two-tailed tests.

\section{Acknowledgments}

The authors thank the members of the Kennedy laboratory for comments on drafts of the manuscript. We also thank Nathalie Marcal, Yi Jiang, Hanna Davies, Maumoud Moussa, and Hanan Elimam for technical assistance. E.W.W. was supported by a Healthy Brains for Healthy Lives (HBHL) scholarship. S.D.G. was supported by postdoctoral fellowships from Fonds de la Recherche Québec - Santé (FRQS) and Canadian Institute for Health Research (CIHR). L.J.T. was supported by scholarships from CIHR, Alzheimer Society of Canada, and Canadian Vascular Network. D.C. was supported by a scholarship from HBHL. The project was supported by grants from CIHR (A.S., E.S.R., E.H., and T.E.K.) and the Alzheimer Society of Canada (T.E.K.). E.S.R. holds a FRQS Research Chair.

Author contributions: Conceptualization: E.W.W., S.D.G. T.E.K.; Methodology: E.W.W., S.D.G., L.J.T.; Investigation: E.W.W., V.R.; Formal analysis: E.W.W., S.D.G., L.J.T.; Writing: E.W.W., S.D.G., T.E.K.; Funding: A.S., E.S.R., E.H., T.E.K.; Resources, S.D.G., D.C.; Supervision: A.S., E.S.R., E.H., T.E.K.

\section{References}

Aida T, Yoshida J, Nomura M, Tanimura A, Iino Y, Soma M, Bai N, Ito Y, Cui W, Aizawa H, et al. 2015. Astroglial glutamate transporter deficiency increases synaptic excitability and leads to pathological repetitive behaviors in mice. Neuropsychopharmacology 40: 1569-1579. doi:10. 1038/npp.2015.26

Bannerman DM, Sprengel R, Sanderson DJ, McHugh SB, Rawlins JN, Monyer H, Seeburg PH. 2014. Hippocampal synaptic plasticity, spatial memory and anxiety. Nat Rev Neurosci 15: 181-192. doi:10.1038/ nrn3677

Boyce R, Glasgow SD, Williams S, Adamantidis A. 2016. Causal evidence for the role of REM sleep theta rhythm in contextual memory consolidation. Science 352: 812-816. doi:10.1126/science.aad5252

Brody DL, Holtzman DM. 2006. Morris water maze search strategy analysis in PDAPP mice before and after experimental traumatic brain injury. Exp Neurol 197: 330-340. doi:10.1016/j.expneurol.2005.10.020

Brun VH, Ytterbø K, Morris RG, Moser MB, Moser EI. 2001. Retrograde amnesia for spatial memory induced by NMDA receptor-mediated long-term potentiation. J Neurosci 21: 356-362. doi:10.1523/ JNEUROSCI.21-01-00356.2001

Buzsáki G. 2002. Theta oscillations in the hippocampus. Neuron 33: 325340. doi:10.1016/S0896-6273(02)00586-X

Cembrowski MS, Wang L, Sugino K, Shields BC, Spruston N. 2016. Hipposeq: a comprehensive RNA-seq database of gene expression in hippocampal principal neurons. eLife 5: e14997. doi:10.7554/eLife. 14997

Clark RE, Martin SJ. 2005. Interrogating rodents regarding their object and spatial memory. Curr Opin Neurobiol 15: 593-598. doi:10.1016/j.conb. 2005.08.014

Crawley JN. 1985. Exploratory behavior models of anxiety in mice. Neurosci Biobehav Rev 9: 37-44. doi:10.1016/0149-7634(85)90030-2

Deacon RM, Rawlins JN. 2006. T-maze alternation in the rodent. Nat Protoc 1: $7-12$. doi:10.1038/nprot.2006.2

D'Hooge R, De Deyn PP. 2001. Applications of the Morris water maze in the study of learning and memory. Brain Res Brain Res Rev 36: 60-90. doi:10. 1016/S0165-0173(01)00067-4

Flores C, Manitt C, Rodaros D, Thompson KM, Rajabi H, Luk KC, Tritsch NX, Sadikot AF, Stewart J, Kennedy TE. 2005. Netrin receptor deficient mice exhibit functional reorganization of dopaminergic systems and do not sensitize to amphetamine. Mol Psychiatry 10: 606-612. doi:10.1038/sj. mp.4001607

Glasgow SD, Labrecque S, Beamish IV, Aufmkolk S, Gibon J, Han D, Harris SN, Dufresne P, Wiseman PW, McKinney RA, et al. 2018. Activity-dependent netrin-1 secretion drives synaptic insertion of GluA1-containing AMPA receptors in the hippocampus. Cell Rep 25: 168-182.e166. doi:10.1016/j.celrep.2018.09.028 
Goldman JS, Ashour MA, Magdesian MH, Tritsch NX, Harris SN, Christofi N, Chemali R, Stern YE, Thompson-Steckel G, Gris P, et al. 2013. Netrin-1 promotes excitatory synaptogenesis between cortical neurons by initiating synapse assembly. J Neurosci 33: 17278-17289. doi:10.1523/ JNEUROSCI.1085-13.2013

Harrison FE, Hosseini AH, McDonald MP. 2009. Endogenous anxiety and stress responses in water maze and Barnes maze spatial memory tasks. Behav Brain Res 198: 247-251. doi:10.1016/j.bbr.2008.10.015

Horn KE, Glasgow SD, Gobert D, Bull SJ, Luk T, Girgis J, Tremblay ME, McEachern D, Bouchard JF, Haber M, et al. 2013. DCC expression by neurons regulates synaptic plasticity in the adult brain. Cell Rep 3: 173185. doi:10.1016/j.celrep.2012.12.005

Incontro S, Díaz-Alonso J, Iafrati J, Vieira M, Asensio CS, Sohal VS, Roche KW, Bender KJ, Nicoll RA. 2018. The CaMKII/NMDA receptor complex controls hippocampal synaptic transmission by kinase-dependent and independent mechanisms. Nat Commun 9: 2069. doi:10.1038/s41467-018-04439-7

Isaac JT, Buchanan KA, Muller RU, Mellor JR. 2009. Hippocampal place cell firing patterns can induce long-term synaptic plasticity in vitro. $J$ Neurosci 29: 6840-6850. doi:10.1523/JNEUROSCI.0731-09.2009

Kalueff AV, Stewart AM, Song C, Berridge KC, Graybiel AM, Fentress JC. 2016. Neurobiology of rodent self-grooming and its value for translational neuroscience. Nat Rev Neurosci 17: 45-59. doi:10.1038/ nrn.2015.8

Kang DS, Yang YR, Lee C, Park B, Park KI, Seo JK, Seo YK, Cho H, Lucio C, Suh PG. 2018. Netrin-1/DCC-mediated PLC $\gamma 1$ activation is required for axon guidance and brain structure development. EMBO Rep 19. e46250. doi:10.15252/embr.201846250

Keino-Masu K, Masu M, Hinck L, Leonardo ED, Chan SS, Culotti JG, Tessier-Lavigne M. 1996. Deleted in colorectal cancer (DCC) encodes a netrin receptor. Cell 87: 175-185. doi:10.1016/S0092-8674(00)81336-7

Kennedy TE, Serafini T, de la Torre JR, Tessier-Lavigne M. 1994. Netrins are diffusible chemotropic factors for commissural axons in the embryonic spinal cord. Cell 78: 425-435. doi:10.1016/0092-8674(94)90421-9

Kim S, Martin KC. 2015. Neuron-wide RNA transport combines with netrinmediated local translation to spatially regulate the synaptic proteome. Elife 4: e04158. doi:10.7554/eLife.04158

Kim JH, Lavan D, Chen N, Flores C, Cooper H, Lawrence AJ. 2013. Netrin-1 receptor-deficient mice show age-specific impairment in drug-induced locomotor hyperactivity but still self-administer methamphetamine. Psychopharmacology 230: 607-616. doi:10.1007/s00213-013-3187-5

Kumaran D, Maguire EA. 2009. Novelty signals: a window into hippocampal information processing. Trends Cogn Sci 13: 47-54. doi:10.1016/j.tics. 2008.11.004

Lai Wing Sun K, Correia JP, Kennedy TE. 2011. Netrins: versatile extracellular cues with diverse functions. Development 138: 2153-2169. doi:10.1242/dev.044529

Louie K, Wilson MA. 2001. Temporally structured replay of awake hippocampal ensemble activity during rapid eye movement sleep. Neuron 29: 145-156. doi:10.1016/S0896-6273(01)00186-6

Lynch G, Larson J, Kelso S, Barrionuevo G, Schottler F. 1983. Intracellular injections of EGTA block induction of hippocampal long-term potentiation. Nature 305: 719-721. doi:10.1038/305719a0

McFarlane HG, Kusek GK, Yang M, Phoenix JL, Bolivar VJ, Crawley JN. 2008. Autism-like behavioral phenotypes in BTBR T+tf/J mice. Genes Brain Behav 7: 152-163. doi:10.1111/j.1601-183X.2007.00330.x

Montgomery SM, Sirota A, Buzsaki G. 2008. Theta and gamma coordination of hippocampal networks during waking and rapid eye movement sleep. J Neurosci 28: 6731-6741. doi:10.1523/JNEUROSCI.1227-08.2008

Morris RG, Garrud P, Rawlins JN, O'Keefe J. 1982. Place navigation impaired in rats with hippocampal lesions. Nature 297: 681-683. doi:10.1038/ 297681a0

Moser EI, Krobert KA, Moser MB, Morris RG. 1998. Impaired spatial learning after saturation of long-term potentiation. Science 281: 2038-2042. doi:10.1126/science.281.5385.2038
Nakazawa K, McHugh TJ, Wilson MA, Tonegawa S. 2004. NMDA receptors, place cells and hippocampal spatial memory. Nat Rev Neurosci 5: 361372. doi:10.1038/nrn1385

O'Keefe J, Dostrovsky J. 1971. The hippocampus as a spatial map. Preliminary evidence from unit activity in the freely-moving rat. Brain Res 34: 171-175. doi:10.1016/0006-8993(71)90358-1

O'Neill J, Senior T, Csicsvari J. 2006. Place-selective firing of CA1 pyramidal cells during sharp wave/ripple network patterns in exploratory behavior. Neuron 49: 143-155. doi:10.1016/j.neuron.2005.10.037

Park P, Sanderson TM, Amici M, Choi SL, Bortolotto ZA, Zhuo M, Kaang BK, Collingridge GL. 2016. Calcium-permeable AMPA receptors mediate the induction of the protein kinase A-dependent component of long-term potentiation in the hippocampus. J Neurosci 36: 622-631. doi:10.1523/ JNEUROSCI.3625-15.2016

Roberts TF, Tschida KA, Klein ME, Mooney R. 2010. Rapid spine stabilization and synaptic enhancement at the onset of behavioural learning. Nature 463: $948-952$. doi: $10.1038 /$ nature 08759

Sadowski JH, Jones MW, Mellor JR. 2016. Sharp-wave ripples orchestrate the induction of synaptic plasticity during reactivation of place cell firing patterns in the hippocampus. Cell Rep 14: 1916-1929. doi:10.1016/j. celrep.2016.01.061

Schindelin J, Arganda-Carreras I, Frise E, Kaynig V, Longair M, Pietzsch T, Preibisch S, Rueden C, Saalfeld S, Schmid B, et al. 2012. Fiji: an open-source platform for biological-image analysis. Nat Methods 9: 676682. doi: $10.1038 /$ nmeth. 2019

Seibenhener ML, Wooten MC. 2015. Use of the Open Field Maze to measure locomotor and anxiety-like behavior in mice. J Vis Exp 96: e52434. doi:10.3791/52434

Serafini T, Kennedy TE, Gaiko MJ, Mirzayan C, Jessell TM, Tessier-Lavigne M. 1994. The netrins define a family of axon outgrowth-promoting proteins homologous to C. elegans UNC-6. Cell 78: 409-424. doi:10. 1016/0092-8674(94)90420-0

Shen K, Cowan CW. 2010. Guidance molecules in synapse formation and plasticity. Cold Spring Harb Perspect Biol 2: a001842. doi:10.1101/ cshperspect.a001842

Silva D, Feng T, Foster DJ. 2015. Trajectory events across hippocampal place cells require previous experience. Nat Neurosci 18: 1772-1779. doi:10. 1038/nn.4151

Silverman JL, Tolu SS, Barkan CL, Crawley JN. 2010. Repetitive self-grooming behavior in the BTBR mouse model of autism is blocked by the mGluR5 antagonist MPEP. Neuropsychopharmacology 35: 976989. doi:10.1038/npp.2009.201

Tong XK, Lecrux C, Rosa-Neto P, Hamel E. 2012. Age-dependent rescue by simvastatin of Alzheimer's disease cerebrovascular and memory deficits. J Neurosci 32: 4705-4715. doi:10.1523/JNEUROSCI.0169-12.2012

Tsien JZ, Chen DF, Gerber D, Tom C, Mercer EH, Anderson DJ, Mayford M, Kandel ER, Tonegawa S. 1996. Subregion- and cell type-restricted gene knockout in mouse brain. Cell 87: 1317-1326. doi:10.1016/S0092-8674 (00)81826-7

Vago DR, Kesner RP. 2008. Disruption of the direct perforant path input to the CA1 subregion of the dorsal hippocampus interferes with spatial working memory and novelty detection. Behav Brain Res 189: 273-283. doi:10.1016/j.bbr.2008.01.002

Vinogradova OS. 2001. Hippocampus as comparator: role of the two input and two output systems of the hippocampus in selection and registration of information. Hippocampus 11: 578-598. doi:10.1002/ hipo. 1073

Vogel-Ciernia A, Wood MA. 2014. Examining object location and object recognition memory in mice. Curr Protoc Neurosci 69: 8.31.1-8.31.17. doi:10.1002/0471142301.ns0831s69

Volenec A, Bhogal RK, Moorman JM, Leslie RA, Flanigan TP. 1997. Differential expression of DCC mRNA in adult rat forebrain. Neuroreport 8: 2913-2917. doi:10.1097/00001756-199709080-00022

Received December 8, 2018; accepted in revised form January 18, 2019. 


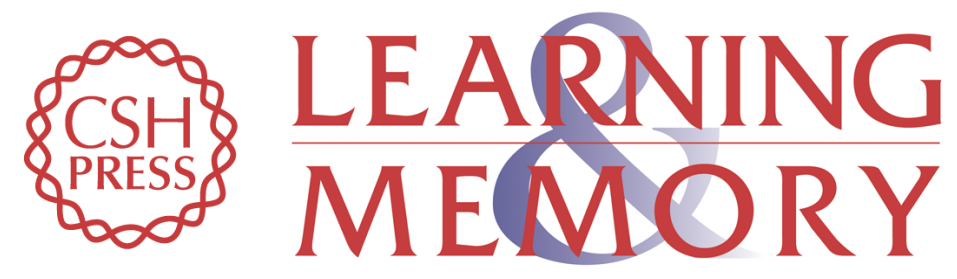

\title{
Spatial memory formation requires netrin-1 expression by neurons in the adult mammalian brain
}

\author{
Edwin W. Wong, Stephen D. Glasgow, Lianne J. Trigiani, et al.
}

Learn. Mem. 2019, 26:

Access the most recent version at doi:10.1101/Im.049072.118

\begin{aligned} & \hline References $\begin{array}{l}\text { This article cites } 50 \text { articles, } 11 \text { of which can be accessed free at: } \\ \text { http://learnmem.cshlp.org/content/26/3/77.full.html\#ref-list-1 }\end{array} \\ & \begin{array}{r}\text { Creative } \\ \text { Commons } \\ \text { License }\end{array} \begin{array}{l}\text { This article is distributed exclusively by Cold Spring Harbor Laboratory Press for the } \\ \text { first } 12 \text { months after the full-issue publication date (see } \\ \text { http://learnmem.cshlp.org/site/misc/terms.xhtml). After } 12 \text { months, it is available under } \\ \text { a Creative Commons License (Attribution-NonCommercial } 4.0 \text { International), as } \\ \text { described at http://creativecommons.org/licenses/by-nc/4.0/. }\end{array} \\ & \begin{array}{c}\text { Receive free email alerts when new articles cite this article - sign up in the box at the } \\ \text { top right corner of the article or click here. }\end{array} \\ & \begin{array}{c}\text { Service } \\ \text { terting }\end{array}\end{aligned}$

